\title{
Allocation of Distributed Flaps for Gust Load Alleviation
}

\author{
Manuel Pusch ${ }^{1}$
}

\begin{abstract}
Increasing the number and functionality of control surfaces provides great potential to improve aircraft performance. This, however, complicates the design of the control allocation system commonly used for flight control. A novel method for linear control allocation is presented which allows considering an arbitrary number of frequency dependent control objectives. The method systematically identifies principal control input directions for predefined performance channels by means of a balancing state space transformation. The effectiveness of the method is proven by designing a gust load alleviation system for a flexible aircraft with distributed trailing edge flaps.
\end{abstract}

\section{INTRODUCTION}

In order to allow for a more economic and environmentally friendly operation of transport aircraft, it is inevitable to actively control, for instance, aerodynamic drag, structural loads or noise emissions [1]. Certainly, the efficiency of each individual control function strongly depends on available control surfaces. Hence, increasing the number of control surfaces and using them for multiple purposes offers great potential to improve aircraft performance [2]. This leads, however, to new challenges in flight control design due to the increased number of control inputs.

In [3], the concept of a variable camber continous trailing edge flap (VCCTEF) is presented. It introduces a large amount of control variables allowing adapting the wing shape according to the current flight conditions. In order to form a smooth trailing edge, virtual control variables based on Fourier series are introduced in [4]. Other approaches are presented in [5] and [6], where Chebyshev and Bernstein polynomials are used respectively for control allocation. Furthermore, the individual segments of each flap allow for active camber control, but are primarily allocated based on simple geometric functions. All in all, for controlling the VCCTEF, linear control allocation using polynomials is generally applied, with the drawback of being independent of any control objective.

On the contrary, common linear control allocation methods, as summarized for example in [7], [8], [9], [10], directly consider the intended control objective. To that end, a so called control effector matrix has to be determined first. In flight control, this matrix typically maps the control surface deflections to the three rotational degrees of freedom of a rigid aircraft. Methods based on inversion or optimization can then be used to determine an appropriate allocation matrix. For controlling the dynamic response of a flexible

\footnotetext{
${ }^{1}$ Manuel Pusch is with the Department of System Dynamics and Control at the German Aerospace Center (DLR), Germany manuel.pusch@dlr. de
}

aircraft, however, a control effector matrix is needed which captures the whole frequency range of interest.

In this paper, a novel linear control allocation method is presented that allows accounting for frequency dependent control objectives. To that end, principal control input directions are identified using balanced truncation. The necessary preliminaries and the proposed procedure for control allocation are described in Section II and Section III, respectively. The effectiveness of the proposed method is then demonstrated with an example of a gust load alleviation (GLA) controller design in Section IV.

\section{PRELIMINARIES}

\section{A. Linear Time-Invariant Systems}

In this paper, the focus is put on allocation of inputs for linear time-invariant (LTI) systems. As described for example in [11], an LTI system

$$
\boldsymbol{G}:\left[\begin{array}{c}
\dot{\boldsymbol{x}}(t) \\
\boldsymbol{w}(t)
\end{array}\right]=\left[\begin{array}{ll}
\boldsymbol{A} & \boldsymbol{B} \\
\boldsymbol{C} & \boldsymbol{D}
\end{array}\right]\left[\begin{array}{l}
\boldsymbol{x}(t) \\
\boldsymbol{r}(t)
\end{array}\right]
$$

with the states $\boldsymbol{x}: \mathbb{R}_{+} \rightarrow \mathbb{R}^{n_{x}}$ maps the input signal $r$ : $\mathbb{R}_{+} \rightarrow \mathbb{R}^{n_{r}}$ to the output signal $\boldsymbol{w}: \mathbb{R}_{+} \rightarrow \mathbb{R}^{n_{w}}$. The system $\boldsymbol{G}$ is defined by the constant matrices $\boldsymbol{A} \in \mathbb{R}^{n_{x} \times n_{x}}, \boldsymbol{B} \in$ $\mathbb{R}^{n_{x} \times n_{r}}, \boldsymbol{C} \in \mathbb{R}^{n_{w} \times n_{x}}, \boldsymbol{D} \in \mathbb{R}^{n_{w} \times n_{r}}$. Furthermore, it is called stable if all eigenvalues of $\boldsymbol{A}$ have negative real part. Note that, for ease of notation, the time argument of signals is commonly dropped.

\section{B. Balanced Order Reduction}

In order to obtain a balanced realization of a stable LTI system $\boldsymbol{G}$, a state transformation $\tilde{\boldsymbol{x}}=\boldsymbol{T} \boldsymbol{x}$ can be found leading to identical controllability and observability Gramians

$$
\boldsymbol{W}_{c}=\boldsymbol{W}_{o}=\operatorname{diag}\left(\sigma_{H, 1}, \ldots, \sigma_{H, n_{x}}\right),
$$

where $\sigma_{H, 1} \geq \ldots \geq \sigma_{H, n_{x}}$ are the Hankel singular values (HSVs), see [12],[13]. The balanced system

$$
\tilde{G}: \quad\left[\begin{array}{c}
\dot{\tilde{x}} \\
\boldsymbol{w}
\end{array}\right]=\left[\begin{array}{cc}
\boldsymbol{T A T ^ { - 1 }} & \boldsymbol{T B} \\
\boldsymbol{C} \boldsymbol{T}^{-1} & D
\end{array}\right]\left[\begin{array}{l}
\tilde{x} \\
\boldsymbol{r}
\end{array}\right]
$$

has the same input-output behavior as $G$, which is dominated by the states $\tilde{\boldsymbol{x}}_{i}$ associated with the largest HSVs $\sigma_{H, i}$. Hence, the balanced state space realization is widely used for model order reduction. A common method for order reduction is to truncate the states with the smallest HSVs by partitioning the state space matrices of $\tilde{G}$ into

$$
\tilde{\boldsymbol{A}}=\left[\begin{array}{ll}
\tilde{\boldsymbol{A}}_{11} & \tilde{\boldsymbol{A}}_{12} \\
\tilde{\boldsymbol{A}}_{21} & \tilde{\boldsymbol{A}}_{11}
\end{array}\right], \tilde{\boldsymbol{B}}=\left[\begin{array}{l}
\tilde{\boldsymbol{B}}_{1} \\
\tilde{\boldsymbol{B}}_{2}
\end{array}\right], \tilde{\boldsymbol{C}}=\left[\begin{array}{ll}
\tilde{\boldsymbol{C}}_{1} & \tilde{\boldsymbol{C}}_{2}
\end{array}\right],
$$


resulting in a reduced order system

$$
\tilde{\boldsymbol{G}}_{1}: \quad\left[\begin{array}{c}
\dot{\tilde{\boldsymbol{x}}}_{1} \\
\boldsymbol{w}
\end{array}\right]=\left[\begin{array}{cc}
\tilde{\boldsymbol{A}}_{11} & \tilde{\boldsymbol{B}}_{1} \\
\tilde{\boldsymbol{C}}_{1} & \boldsymbol{D}
\end{array}\right]\left[\begin{array}{c}
\tilde{\boldsymbol{x}}_{1} \\
\boldsymbol{r}
\end{array}\right] .
$$

An alternative method would be to residualize the states with the smallest HSVs, which allows retaining the steady state behavior of the system. Furthermore, a certain frequency or time interval may be emphasized for a balanced order reduction as described in [14].

\section{C. $H_{\infty}$ Control Design}

In this paper, the $H_{\infty}$-framework [15] is used for control design. To that end, the generalized plant

$$
\boldsymbol{P}: \quad\left[\begin{array}{c}
\dot{\boldsymbol{x}} \\
\boldsymbol{e} \\
\boldsymbol{y}
\end{array}\right]=\left[\begin{array}{ccc}
\boldsymbol{A} & \boldsymbol{B}_{d} & \boldsymbol{B}_{u} \\
\boldsymbol{C}_{e} & \boldsymbol{D}_{e d} & \boldsymbol{D}_{e u} \\
\boldsymbol{C}_{y} & \boldsymbol{D}_{y d} & \boldsymbol{D}_{y u}
\end{array}\right]\left[\begin{array}{l}
\boldsymbol{x} \\
\boldsymbol{d} \\
\boldsymbol{u}
\end{array}\right]
$$

is defined, which maps the performance and control inputs $\boldsymbol{d}$ and $\boldsymbol{u}$ to the performance and measurement outputs $\boldsymbol{e}$ and $\boldsymbol{y}$. A stabilizing controller $\boldsymbol{K}$ is then derived that minimizes an upper bound $\gamma$ on the $H_{\infty}$-norm

$$
\left\|\mathcal{F}_{l}(\boldsymbol{P}, \boldsymbol{K})\right\|_{\infty}=\sup _{\boldsymbol{d} \in \mathcal{L}_{2} \backslash\{\mathbf{0}\}} \frac{\|e\|_{2}}{\|d\|_{2}}<\gamma
$$

of the closed-loop interconnection given by the lower fractional transformation $\mathcal{F}_{l}(\boldsymbol{P}, \boldsymbol{K})$ depicted Figure 1. In order to achieve the desired closed-loop performance, weighting filters are typically added to the performance channels $\boldsymbol{d}$ and $e$, introducing some additional degrees of freedom for controller tuning.

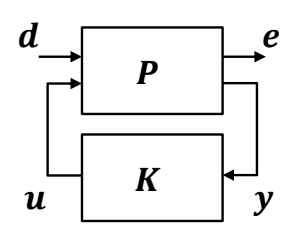

Fig. 1. Closed-loop interconnection $\mathcal{F}_{l}(\boldsymbol{P}, \boldsymbol{K})$.

\section{LINEAR ALLOCATION}

In order to shorten notations, only the allocation of inputs, generally known as control allocation, is treated here. However, the allocation of outputs, also called sensor allocation, may be derived in a similar way.

\section{A. Linear Control Allocation Problem}

In this section, the linear control allocation problem in terms of input directions is discussed. Generally, linear control allocation is described by

$$
\boldsymbol{u}=\boldsymbol{T}_{u v} \boldsymbol{v}
$$

where $\boldsymbol{T}_{u v} \in \mathbb{R}^{n_{u} \times n_{v}}$ with $n_{v}<n_{u}$ is the allocation matrix mapping the virtual control inputs $\boldsymbol{v} \in \mathbb{R}^{n_{v}}$, to the actual control inputs $\boldsymbol{u} \in \mathbb{R}^{n_{u}}$. The allocation matrix

$$
\boldsymbol{T}_{u v}=\left[\begin{array}{lll}
k_{1} \tilde{\boldsymbol{v}}_{1} & \ldots & k_{n_{v}} \tilde{\boldsymbol{v}}_{n_{v}}
\end{array}\right]
$$

contains $n_{v}$ linear independent control input directions $\tilde{\boldsymbol{v}}_{i}$ with $\left|\tilde{\boldsymbol{v}}_{i}\right|=1$ for $i=1, \ldots, n_{v}$, which are scaled by a constant factor $k_{i} \in \mathbb{R} \backslash\{0\}$. Obviously, control allocation can lead to a degradation of controllability as certain control input directions may lie within the null space of $\boldsymbol{T}_{u v}$. Hence, it is of major interest that the control allocation matrix contains the principal input directions in order to achieve a certain control objective. The linear control allocation problem considered here is thus partitioned into

1) finding a minimum number of linear independent control input directions $\tilde{\boldsymbol{v}}_{i}$ which have high impact on the predefined control objective, and

2) finding appropriate scaling factors $k_{i}$ for each control input direction.

Note that, the determination of the scaling factors may be neglected at this point as it can also be seen as a part of the subsequent controller design. For numerical stability, however, it makes sense to scale each virtual input so the corresponding time signals are of comparable magnitude.

\section{B. Linear Control Allocation using Balanced Truncation}

In this section, a novel method for designing a linear control allocation matrix $\boldsymbol{T}_{u v}$ is proposed. The procedure starts with the generalized plant $\boldsymbol{P}$ from Equation (6), which is assumed to be fully controllable by $\boldsymbol{u}$ and fully observable by $\boldsymbol{y}$. In a first step, an auxiliary balanced truncation is performed on the open-loop performance subsystem

$$
\boldsymbol{P}_{d \rightarrow e}:\left[\begin{array}{c}
\dot{\boldsymbol{x}} \\
\boldsymbol{e}
\end{array}\right]=\left[\begin{array}{cc}
\boldsymbol{A} & \boldsymbol{B}_{d} \\
\boldsymbol{C}_{e} & \boldsymbol{D}_{e d}
\end{array}\right]\left[\begin{array}{l}
\boldsymbol{x} \\
\boldsymbol{d}
\end{array}\right]
$$

of $\boldsymbol{P}$ using the balancing state transformation $\tilde{\boldsymbol{x}}=\boldsymbol{T} \boldsymbol{x}$. The reduced performance subsystem has the states $\tilde{\boldsymbol{x}}_{1} \in \mathbb{R}^{n_{\tilde{x}_{1}}}$ associated to the largest HSVs $\sigma_{H, i}>\sigma_{H, \min }$ of $\boldsymbol{P}_{d \rightarrow e}$. Obviously, a desired order $n_{\tilde{x}_{1}}$ of the reduced performance subsystem is determined by adjusting the threshold $\sigma_{H \text {,min }}$. Note that, balanced truncation does not necessarily preserve pole locations, which may lead to an undesired transformation of system dynamics. However, for systems with weak natural damping (e.g. lightweight structures), a good matching is typically achieved, see [16], [17]. Hence, special care has to be taken in case the performance subsystem is dominated by poles with high natural damping in the frequency range of interest.

Secondly, the control input matrix $\boldsymbol{B}_{u}$ of the generalized plant $\boldsymbol{P}$ is transformed to

$$
\tilde{\boldsymbol{B}}_{u}=\boldsymbol{T} \boldsymbol{B}_{u}=\left[\begin{array}{c}
\tilde{\boldsymbol{B}}_{u, 1} \\
\tilde{\boldsymbol{B}}_{u, 2}
\end{array}\right]
$$

In Equation (11), the submatrix $\tilde{\boldsymbol{B}}_{u, 1} \in \mathbb{R}^{n_{\tilde{x}_{1}} \times n_{u}}$ is associated to the dominating states $\tilde{\boldsymbol{x}}_{1}$ of $\boldsymbol{P}_{d \rightarrow e}$. Hence, the input directions of $\tilde{\boldsymbol{B}}_{u, 1}$ are considered as best suited for control allocation.

Thirdly, the input directions of $\tilde{\boldsymbol{B}}_{u, 1}$ are orthogonalized 
using singular value decomposition (SVD). This leads to

$$
\begin{aligned}
\tilde{\boldsymbol{B}}_{u, 1} & =\tilde{\boldsymbol{U}} \tilde{\boldsymbol{\Sigma}} \tilde{\boldsymbol{V}}^{T} \\
& =\left[\begin{array}{ll}
\tilde{\boldsymbol{U}}_{1} & \tilde{\boldsymbol{U}}_{2}
\end{array}\right]\left[\begin{array}{cc}
\tilde{\boldsymbol{\Sigma}}_{1} & 0 \\
0 & \tilde{\boldsymbol{\Sigma}}_{2}
\end{array}\right]\left[\begin{array}{cc}
\tilde{\boldsymbol{V}}_{1}^{T} & \tilde{\boldsymbol{V}}_{2}^{T}
\end{array}\right]^{T},
\end{aligned}
$$

where $\tilde{\boldsymbol{U}} \in \mathbb{R}^{n_{\tilde{x}_{1} \times n_{\tilde{x}_{1}}}}$ and $\tilde{\boldsymbol{V}}^{T} \in \mathbb{R}^{n_{u} \times n_{u}}$ are unitary matrices. Besides, the rectangular diagonal matrix $\tilde{\boldsymbol{\Sigma}} \in$ $\mathbb{R}^{n_{\tilde{x}_{1}} \times n_{u}}$ lists the singular values $\tilde{\sigma}_{i} \geq 0$ for $i=1, \ldots, n_{\tilde{\sigma}}$, $n_{\tilde{\sigma}}=\min \left(n_{\tilde{x}_{1}}, n_{u}\right)$ on its diagonal. Now, the columns of matrix $\tilde{\boldsymbol{V}}_{1}^{T}=\left[\begin{array}{lll}\tilde{\boldsymbol{v}}_{1} & \ldots & \tilde{\boldsymbol{v}}_{n_{v}}\end{array}\right]$ associated to the singular values $\tilde{\sigma}_{i}>\tilde{\sigma}_{\text {min }} \geq 0$ are considered as the principal control input directions. Thus, the vectors $\tilde{\boldsymbol{v}}_{i}, i=1, . ., n_{v}$ are directly used to define the virtual control inputs $\boldsymbol{v}$. In general, a minimum number of virtual control inputs is desired which still allows sufficient controllability in the frequency range of interest. To that end, the computed HSVs and singular values can be used to appropriately balance complexity (in terms of control inputs) and controllability.

In a fourth and final step, the principal control input directions $\tilde{\boldsymbol{v}}_{i}$ are scaled by the inverse of the corresponding singular values. This leads to the control allocation matrix

$$
\boldsymbol{T}_{u v}=\tilde{\boldsymbol{V}}_{1}^{T} \tilde{\boldsymbol{\Sigma}}_{1}^{-1}=\left[\begin{array}{lll}
\frac{1}{\tilde{\sigma}_{1}} \tilde{\boldsymbol{v}}_{1} & \cdots & \frac{1}{\tilde{\sigma}_{n_{v}}} \tilde{\boldsymbol{v}}_{n_{v}}
\end{array}\right],
$$

where $\tilde{\boldsymbol{\Sigma}}_{1}$ is a square matrix, see also Equation (12). Furthermore, it may be advantageous to scale the allocation matrix $\boldsymbol{T}_{u v}$ by its induced 1-norm $\left\|\boldsymbol{T}_{u v}\right\|_{i 1}$ to guarantee a bounded control input $\|\boldsymbol{u}\|_{\infty} \leq 1$ for a bounded virtual input $\|\boldsymbol{v}\|_{\infty} \leq 1$.

Summing up, the four steps required to design the control allocation matrix are

1) balanced truncation of $\boldsymbol{P}_{d \rightarrow e}$ with $\sigma_{H, \min _{\tilde{\boldsymbol{B}}}} \rightarrow \boldsymbol{T}$

2) transformation and truncation of $\boldsymbol{B}_{u} \rightarrow \tilde{\boldsymbol{B}}_{u, 1}$

3) SVD of $\tilde{\boldsymbol{B}}_{u, 1}$ with $\tilde{\sigma}_{\min } \rightarrow \tilde{\boldsymbol{V}}_{1}^{T}$

4) scaling of $\tilde{\boldsymbol{V}}_{1}^{T} \rightarrow \boldsymbol{T}_{u v}$.

In the proposed procedure, the thresholds $\sigma_{H, \min }$ and $\tilde{\sigma}_{\min }$ can be used as tuning knobs for designing the control allocation matrix $\boldsymbol{T}_{u v}$. In addition to that, an initial scaling of inputs and outputs of the generalized plant $\boldsymbol{P}$ also affects the resulting control allocation matrix and may be used to balance certain performance specifications.

\section{EXAMPLE: GUST LOAD ALLEVIATION}

For the evaluation of the effectiveness of the proposed allocation method, a GLA system is designed for a flexible aircraft with distributed flaps. To that end, the distributed flap inputs are first allocated using the method proposed in Section III-B. Subsequently, an $H_{\infty}$ output feedback controller is designed to alleviate structural loads during gust encounter. As a performance measure, the reduction of the peaks of the wing root bending and torsional moment is considered. The achieved performance is then compared with the performance of a GLA system using Chebyshev polynomials for control allocation and a GLA system without any allocation.

\section{A. Modeling Flexible Aircraft}

The flexible aircraft used for simulations is based on the Digital-X project [18] and modeled according to [19]. The aeroservoelastic model interconnects a finite element model of the airframe with an unsteady aerodynamics model. The aerodynamics model is computed in frequency domain by means of the doublet lattice method [20] and transformed to time domain using Roger's rational function approximation [21]. For the gust input, the aircraft is divided into 25 zones in the direction of flight and a Padé approximation is used to model the time delay for each zone. As control surfaces, a pair of elevators and 9 equally distributed trailing edge flaps on each wing are modeled (see also Figure 2). The actuators for each control surface are approximated by a first order low pass filter with a bandwidth of $\omega_{c}=20 \mathrm{rad} / \mathrm{s}$. Eventually, the resulting structural loads are then recovered by the force summation method [22]. More details on aeroservoelastic modeling can be found in [23], [20], [19].

For evaluation of the proposed control allocation method, one single flight point at $M a=0.86$ and $h=9108 \mathrm{~m}$ is considered. Furthermore, only symmetric excitations are taken into account, meaning that gust encounter as well as control surface deflections are assumed to be equal on the left and right hand side of the aircraft. Hence, the model order can be reduced by discarding all non-symmetric modes and by combining the actuator models on both sides.

The nonlinear aircraft model is linearized around steady horizontal flight. In addition to that, the order of the linearized aircraft is further reduced to a number of 44 states by truncation in modal and balanced coordinates. The resulting reduced order LTI system has 1 vertical gust and 10 control surface inputs. As outputs, acceleration sensors at the wing tip and the center of gravity as well as the wing root bending and torsional moment are taken into account. Due to the symmetric excitations, the outputs also are assumed to be equal on both sides of the aircraft and hence are not determined twice.

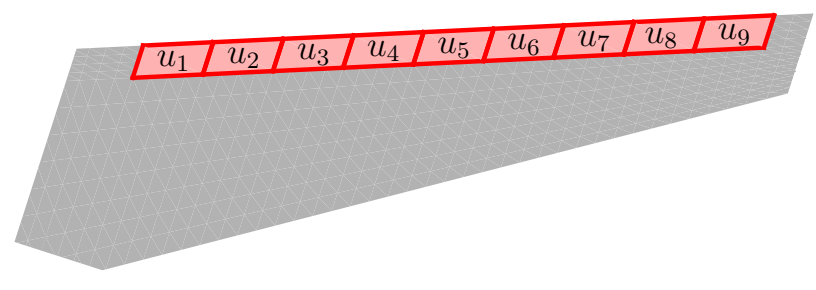

Fig. 2. Distributed trailing edge flaps on the wing used for GLA.

\section{B. Allocation of Distributed Flaps}

Before designing the actual GLA system, the distributed trailing edge flaps are allocated according to the method proposed in Section III-B.

In a first step, the open-loop performance subsystem $\boldsymbol{P}_{d \rightarrow e}$ from the gust input to the loads outputs is selected and a balancing state space transformation is computed. The resulting states are ordered according to their HSVs, see also Figure 3. 
For the second step, the threshold $\sigma_{H \text {,min }}$ is chosen so that only the four most dominant states remain after truncation. In Figure 4, the frequency responses of the performance subsystem with full and reduced order are compared. It can be seen that the main dynamics from the gust input to the loads outputs are captured well.

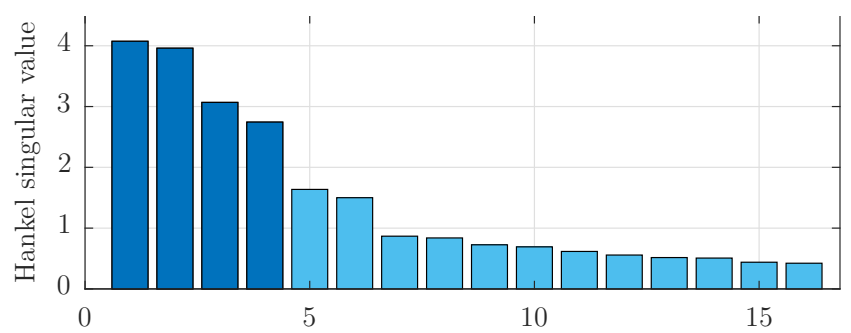

Fig. 3. Largest HSVs of $\boldsymbol{P}_{d \rightarrow e}$ with the HSVs remaining after truncation marked in dark.

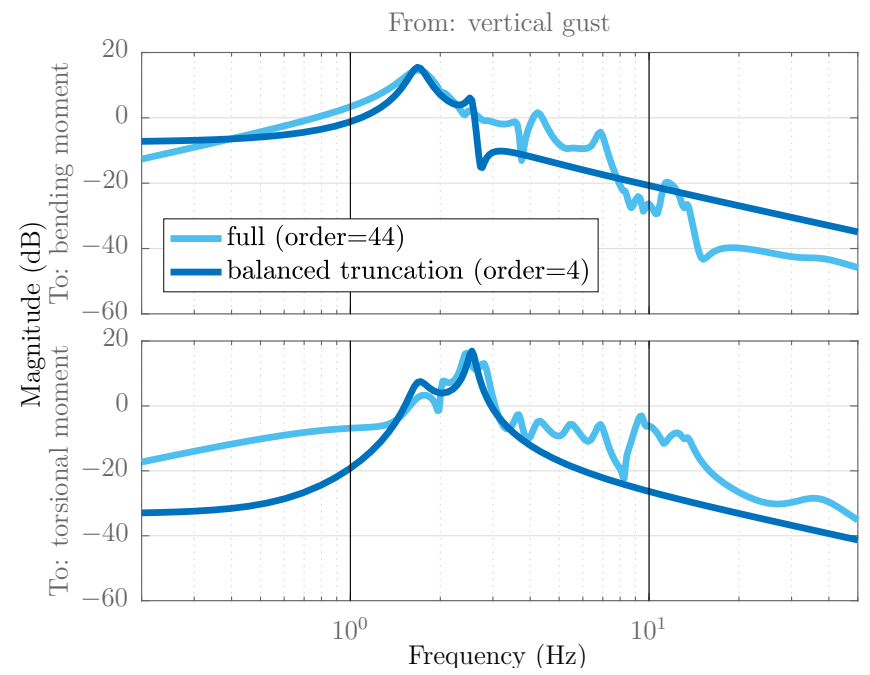

Fig. 4. Frequency response magnitude of $\boldsymbol{P}_{d \rightarrow e}$ with full and reduced order.

In the third step, an SVD is carried out on the remaining control input matrix $\tilde{\boldsymbol{B}}_{u, 1}$. The resulting singular values are plotted in Figure 5 (left), where two of the singular values are considerably smaller than the others. Hence, they are truncated by adjusting the threshold $\tilde{\sigma}_{\min }$. The remaining

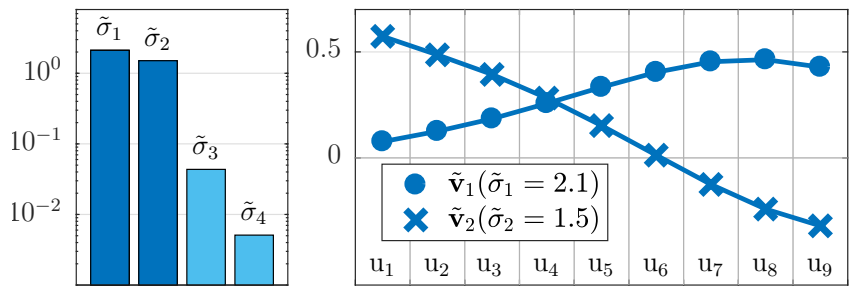

Fig. 5. Singular values $\tilde{\sigma}_{i}$ and principal input directions $\tilde{\boldsymbol{v}}_{i}$ of $\tilde{\boldsymbol{B}}_{u, 1}$, where $\tilde{\sigma}_{3}$ and $\tilde{\sigma}_{4}$ are truncated.

singular values correspond to the selected principal control input directions plotted in Figure 5 (right). A physical interpretation of these two directions is difficult due to numerous interfering aeroelastic effects. On the one hand, the spanand chordwise distance of the respective flaps to the wing root plays an important role. On the other hand, natural coupling of bending and torsion as well as reversal effects of flap deflections for a swept flexible wing may influence the results. Nevertheless, $\tilde{\boldsymbol{v}}_{1}$ seems to be well suited to control the wing bending as it causes larger deflections at the outer wing. On the contrary, the control surface deflections caused by $\tilde{\boldsymbol{v}}_{2}$ are larger at the inner part of the wing, where an increased chord length allows a better control of the wing root torsional moment.

In the fourth and final step, the selected principal control input directions are scaled with the inverse of the corresponding singular values. This results in the control allocation matrix $\boldsymbol{T}_{u v}$, which allocates the trailing edge flaps according to the predefined control objective. However, by deflecting the flaps on the wing for the purpose of GLA, some pitching moment is induced, which is typically compensated using the elevators. Hence, an additional column is added to the control allocation matrix which allows directly controlling elevator deflections. The resulting augmented control allocation matrix $\tilde{\boldsymbol{T}}_{u v}$ is then used to design an GLA controller as described in the next section.

\section{Gust Load Alleviation Controller Design}

For evaluation of the proposed control allocation method, a GLA controller is designed according to the $H_{\infty}$ control methodology described in Section II-C. To that end, the flexible aircraft model from Section IV-A is augmented by weighted inputs and outputs enforcing the required performance specifications. Additionally, the augmented control allocation matrix $\tilde{\boldsymbol{T}}_{u v}$ is added, resulting in the generalized plant $\boldsymbol{P}$ marked with a dashed line in Figure 6.

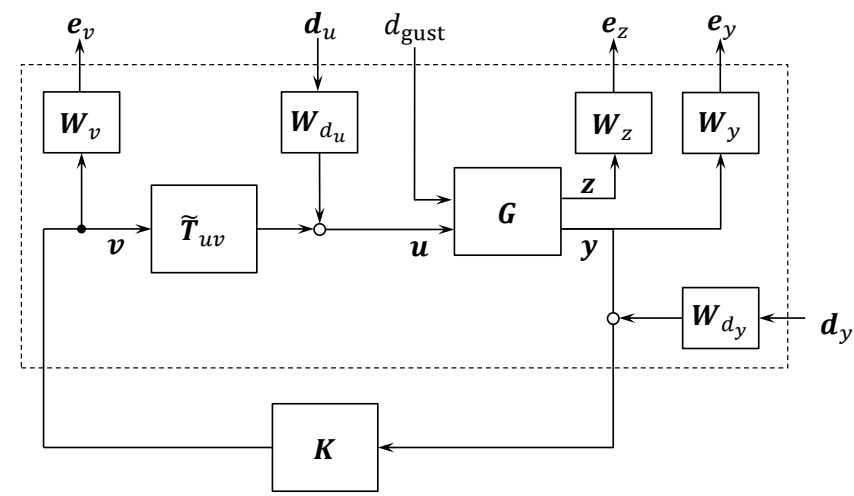

Fig. 6. Closed-loop interconnection for GLA controller design, where the generalized plant $\boldsymbol{P}$ is encircled with a dashed line.

As it can be seen, the outputs of the plant $G$ are divided into measurable outputs $\boldsymbol{y}$ and non-measurable outputs $\boldsymbol{z}$. Certainly, the two acceleration measurements described above are collected in $\boldsymbol{y}$, whereas $\boldsymbol{z}$ consists of the nonmeasurable wing root bending and torsional moments. Both vector signals are weighted, yielding the performance outputs $\boldsymbol{e}_{z}$ and $\boldsymbol{e}_{y}$. For the measurement outputs, a constant 
weighting matrix $\boldsymbol{W}_{y}=0.1 \boldsymbol{I}_{n_{y}}$ is employed. For the loads outputs, the bandpass weighting filters

$$
\boldsymbol{W}_{z_{i}}(s)=c_{z_{i}} \frac{0.001 s^{2}+56.55 s+0.3948}{s^{2}+56.55 s+394.8}
$$

for $i=1, \ldots, n_{z}$ are chosen, where the gains $\boldsymbol{c}_{z}=$ $\left[\begin{array}{lll}c_{z_{1}} & \ldots & c_{z_{n_{z}}}\end{array}\right]^{T}$ are subject to be tuned. In order to limit the control effort, the virtual control inputs are weighted by the bandstop filters

$$
\boldsymbol{W}_{v_{i}}(s)=c_{v_{i}} \frac{s^{2}+56.55 s+394.8}{0.001 s^{2}+56.55 s+0.3948}
$$

with $i=1, \ldots, n_{v}$ and the tuning parameters $\boldsymbol{c}_{v}=\left[\begin{array}{lll}c_{v_{1}} & \ldots & c_{v_{n_{v}}}\end{array}\right]^{T}$. The weighting filters $\boldsymbol{W}_{z}=$ $\operatorname{diag}\left(\boldsymbol{W}_{z_{1}}, \ldots, \boldsymbol{W}_{z_{n_{z}}}\right)$ and $\boldsymbol{W}_{v}=\operatorname{diag}\left(\boldsymbol{W}_{v_{1}}, \ldots, \boldsymbol{W}_{v_{n_{v}}}\right)$ thus restrict control activity and performance requirements to the frequency range of interest between $1 \mathrm{~Hz}$ and $10 \mathrm{~Hz}$. Each weighting filter adds two additional states to the generalized plant and introduces a degree of freedom for controller tuning. Hence, the number of states as well as the number of tuning parameters can be reduced efficiently by weighting the virtual control inputs $v$ instead of the real control inputs $\boldsymbol{u}$.

As performance inputs, the symmetric gust input $d_{\text {gust }}$, the control input disturbance $\boldsymbol{d}_{u}$ and the measurement noise $\boldsymbol{d}_{y}$ are collected in $\boldsymbol{d}=\left[\begin{array}{lll}d_{\text {gust }} & \boldsymbol{d}_{u}^{T} & \boldsymbol{d}_{y}^{T}\end{array}\right]^{T}$. The corresponding input weights $\boldsymbol{W}_{d_{u}}$ and $\boldsymbol{W}_{d_{y}}$ are set to a constant gain of 0.1 for each signal.

The $H_{\infty}$ optimal controller $\boldsymbol{K}$ is then synthesized by solving two Riccati equations using the Robust Control Toolbox of Matlab [24]. The actual GLA controller

$$
\boldsymbol{K}_{\mathrm{GLA}}=\tilde{\boldsymbol{T}}_{u v} \boldsymbol{K}
$$

is finally obtained by multiplying the virtual control outputs of $\boldsymbol{K}$ with the augmented control allocation matrix $\tilde{\boldsymbol{T}}_{u v}$.

\section{Tuning Setup}

In order to obtain a highly performant GLA system, it needs to be tuned. To that end, closed-loop time domain simulations are performed for a "1-cos" gust excitation with a gust gradient distance of $350 \mathrm{ft}$, see also [25]. The increments of the resulting loads and control surface deflections are then optimized by adjusting the weighting filter gains $\boldsymbol{c}_{z}$ and $\boldsymbol{c}_{v}$. After all, the optimization setup is defined as

$$
\begin{array}{ll}
\underset{\boldsymbol{c}_{v}, \boldsymbol{c}_{z}}{\operatorname{minimize}} & \max \left(\bar{M}_{\text {bending }}, \bar{M}_{\text {torsion }}\right) \\
\text { subject to } & \left|u_{i}\right| \leq 25^{\circ}, i=1, \ldots, 10,
\end{array}
$$

where $\bar{M}_{\text {bending }}$ and $\bar{M}_{\text {torsion }}$ are the normalized wing root bending respectively torsional moment increments. Eventually, the optimization is performed in MOPS [26] using an extended version of the pattern search algorithm proposed in [27].

\section{E. Results}

To assess the performance of the proposed allocation method, three different control approaches for GLA are compared. In the first approach ("Chebyshev"), the trailing edge flaps are allocated using Chebyshev polynomials of the first kind. Using two virtual control inputs $v_{1}$ and $v_{2}$, a single flap deflection is then described by

$$
u_{i}=v_{1}+\frac{n_{\text {flap }}-i}{n_{\text {flap }}-1} v_{2}, i=1, \ldots, n_{\text {flap }},
$$

where the number of trailing edge flaps $n_{\text {flap }}=9$. Note that the control input of the elevators is handled separately here as well (see Section IV-B). In the second control approach ("Balanced") the control allocation matrix determined in Section IV-B is used, which is based on an auxiliary balanced truncation. And for the third approach ("All Flaps"), the allocation matrix $\tilde{\boldsymbol{T}}_{u v}=\boldsymbol{I}_{n_{u}}$, meaning that all flaps are directly controlled and no allocation is applied.

The resulting GLA performance, quantified by the achieved reduction of the peak loads, is good for all three control approaches as it is shown in Figure 7. Furthermore,

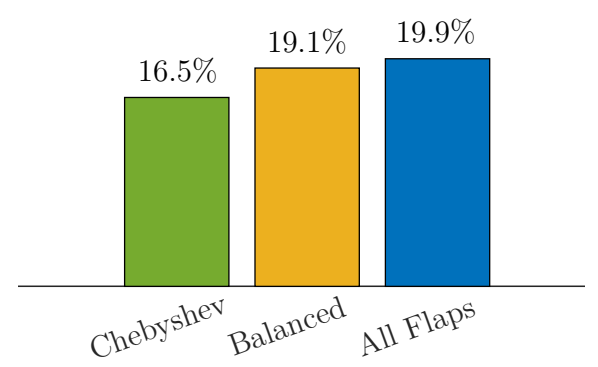

Fig. 7. Comparison of achieved load reduction performance.

it can be seen that the "Balanced" approach outperforms the "Chebyshev" approach by 2.6 percentage points. This means that the proposed allocation method allows a considerably increased GLA performance by directly taking into account the control objectives. In addition to that, the loads reduction is only marginally better using the controller designed without any control allocation. As this approach ("All Flaps") is considered as an upper bound on the achievable GLA performance, the "Balanced" approach is already close to this upper bound, but requires a clearly lower flap deflections as depicted in Figure 8.

From a practical point of view, also numerical stability plays an important role for controller design. For many synthesis methods, numerical issues arise when the size of the control problem is too big. Hence, it is usually of advantage to keep the number of states, inputs and outputs at a minimum in order to be able to find appropriate solutions for controller candidates and tune them accordingly. For the example given here, this can also be seen in Table I, where an increased number of virtual control inputs $n_{v}$ leads to an increased computational effort for controller synthesis and tuning. The reason for this is that weighting filters are assigned to each virtual control input, adding additional states and tuning parameters to the generalized 


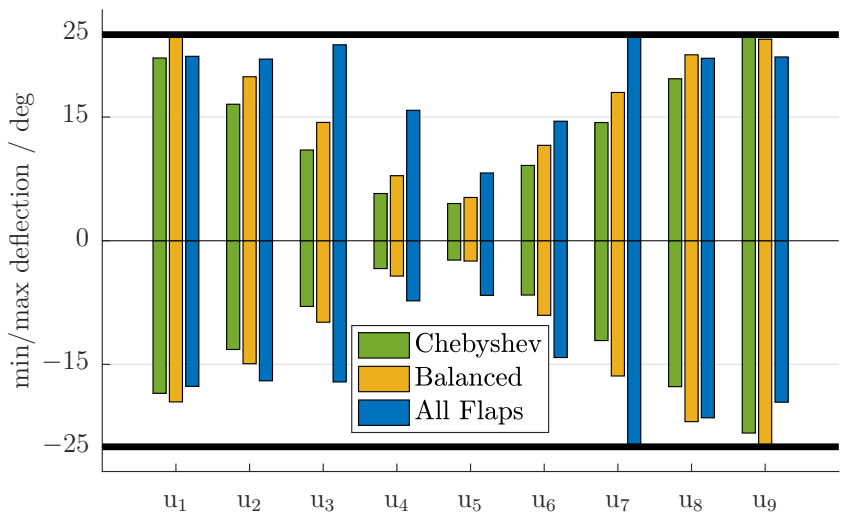

Fig. 8. Comparison of minimum and maximum trailing edge flap deflections.

plant. Note that the given computation times have to be seen as relative measures as controller design takes place under simplified assumptions here. Nevertheless, the size of the control problem does not only affect computational effort but also convergence of the optimization. Thus, a control allocation system which reduces the number of control inputs and keeps performance losses at a minimum offers great practical benefits.

\begin{tabular}{l|c|c|c} 
& Chebyshev & Balanced & All Flaps \\
\hline$n_{v}$ (incl. elevators) & 3 & 3 & 10 \\
order of $\boldsymbol{P}$ & 54 & 54 & 68 \\
\hline synthesis time / ms & 53.8 & 56.4 & 80.9 \\
tuning time / min & 10.2 & 9.9 & 35.5
\end{tabular}

TABLE I

COMPARISON OF CONTROL PROBLEM SIZE AND COMPUTATION TIMES

\section{CONCLUSIONS}

A novel method for linear control allocation is presented that allows to consider frequency dependent control objectives. To that end, principal control input directions are identified using HSVs as well as singular values. The proposed allocation method can be applied to systems with a large number of control inputs and may also be used for sensor allocation. The effectiveness of the proposed method is evaluated by designing a GLA system for a flexible aircraft with distributed flaps. The achieved load reduction is considerably higher than using Chebyshev polynomials for control allocation. Furthermore, a GLA controller which is designed without any control allocation performs only marginally better while requiring larger control surface deflections. Hence, the proposed allocation method shows high potential by greatly simplifying controller design and tuning while allowing to achieve a comparable closed-loop performance.

\section{ACKNOWLEDGMENT}

The author thanks Thiemo Kier, Andreas Knoblach and Julian Theis for their insights and helpful discussions. This work was founded in the frame of the German Aeronautical Research Program project "Atlas ${ }^{2}$ Hybrid", grant number 20A1303B.

\section{REFERENCES}

[1] International Energy Agency. Transport, Energy and $\mathrm{CO}_{2}, 2009$.

[2] Daniel Reckzeh. Multifunctional wing moveables design of the A350XWB and the way to future concepts. In 29th Congress of the International Council of the Aeronautical Sciences (ICAS), St. Petersburg, ISBN, pages 3-932182, 2014.

[3] Nhan Nguyen. Elastically shaped future air vehicle concept. NASA Innovation Fund Award, 2010.

[4] Nhan Nguyen and James Urnes. Aeroelastic modeling of elastically shaped aircraft concept via wing shaping control for drag reduction. In AIAA Atmospheric Flight Mechanics Conference, page 4642, 2012.

[5] Jovan Boskovic, Richard Wise, and Joseph A. Jackson. A flutter suppression and drag optimization approach for flexible aircraft. In 58th AIAA/ASCE/AHS/ASC Structures, Structural Dynamics, and Materials Conference. American Institute of Aeronautics and Astronautics (AIAA), jan 2017.

[6] David L Rodriguez, Michael J Aftosmis, Marian Nemec, and George R Anderson. Optimization of flexible wings with distributed flaps at offdesign conditions. Journal of Aircraft, 53(6):1731-1745, 2016.

[7] Michael W Oppenheimer, David B Doman, and Michael A Bolender. Control allocation. In The Control Handbook, Second Edition: Control System Applications, Second Edition, pages 8-1. CRC Press, 2010.

[8] Tor A Johansen and Thor I Fossen. Control allocation - a survey. Automatica, 49(5):1087-1103, 2013.

[9] Wayne Durham, Kenneth A Bordignon, and Roger Beck. Aircraft Control Allocation. John Wiley \& Sons, 2016.

[10] Marc Bodson. Evaluation of optimization methods for control allocation. Journal of Guidance, Control, and Dynamics, 25(4):703-711, 2002.

[11] Thomas Kailath. Linear systems, volume 156. Prentice-Hall Englewood Cliffs, NJ, 1980.

[12] Bruce Moore. Principal component analysis in linear systems: Controllability, observability, and model reduction. IEEE transactions on automatic control, 26(1):17-32, 1981.

[13] Alanj Laub, Michelt Heath, C Paige, and R Ward. Computation of system balancing transformations and other applications of simultaneous diagonalization algorithms. IEEE Transactions on Automatic Control, 32(2):115-122, 1987.

[14] Wodek Gawronski and Jer-Nan Juang. Model reduction in limited time and frequency intervals. International Journal of Systems Science, 21(2):349-376, 1990.

[15] Sigurd Skogestad and Ian Postlethwaite. Multivariable feedback control: analysis and design, volume 2. Wiley New York, 2007.

[16] C. Z. Gregory. Reduction of large flexible spacecraft models using internal balancing theory. Journal of Guidance, Control, and Dynamics, 7(6):725-732, nov 1984.

[17] Wodek Gawronski. Balanced control of flexible structures, volume 211. Springer, 1996.

[18] N Kroll and C Rossow. Digital-X: DLRs way towards the virtual aircraft. In NIA CFD Conference, pages 6-8, 2012.

[19] Thiemo Kier. An integrated loads analysis model including unsteady aerodynamic effects for position and attitude dependent gust fields. In International Forum on Aeroelasticity and Structural Dynamics, 2011.

[20] W.P. Rodden and E.H. Johnson. MSC.Nastran Version 68, Aeroelastic Analysis and User's Guide, 2004.

[21] Kenneth L. Roger. Airplane math modelling methods for active control design. Structures and Materials Panel, 1977.

[22] R. Bisplinghoff, H. Ashley, and R. Halfman. Aeroelasticity, 1955.

[23] Frederic M. Hoblit. Gust loads on aircraft: concepts and applications. AIAA, 1988.

[24] Gary Balas, Richard Chiang, Andy Packard, and Michael Safonov. Robust control toolbox user's guide R2016b. MathWorks, 3, 2016.

[25] Federal Aviation Administration. Federal Aviation Regulations Part 25, Airworthiness Standards: Transport Category, 2015.

[26] Hans-Dieter Joos. A multiobjective optimisation-based software environment for control systems design. In Computer Aided Control System Design, 2002. Proceedings. 2002 IEEE International Symposium on, pages 7-14. IEEE, 2002.

[27] Robert Hooke and Terry A Jeeves. "Direct Search" solution of numerical and statistical problems. Journal of the ACM (JACM), 8(2):212-229, 1961. 